PROCEEDINGS OF THE

AMERICAN MATHEMATICAL SOCIETY

Volume 130, Number 5, Pages 1407-1412

S 0002-9939(01)06333-X

Article electronically published on October 5, 2001

\title{
SEMIGROUPS OF MAPPINGS WITH RIGID LIPSCHITZ CONSTANT
}

\author{
ENRIQUE LLORENS-FUSTER
}

(Communicated by Jonathan M. Borwein)

\begin{abstract}
It is shown that two well-known uniformly fixed point free lipschitzian semigroups of mappings have minimal Lipschitz constant on the positive part of the unit ball of $\ell_{2}$. This implies that a question raised by T. Kuczumow has a negative answer.
\end{abstract}

\section{INTRODUCTION}

Let $(X,\|\cdot\|)$ be a Banach space, and let $C$ be a bounded closed convex subset of $X$. A map $T: C \rightarrow X$ is $k$-lipschitzian on $C$ whenever $\|T(x)-T(y)\| \leq k\|x-y\|$ for all $x, y \in C$. When $k=1, T$ is also called nonexpansive.

A mapping $T: C \rightarrow C$ is uniformly k-lipschitzian whenever each member of the semigroup of mappings $\left\{T^{n}: n \in \mathbb{N}\right\}$ admits the Lipschitz constant $k$.

Since at least 1973 (for instance in G-K-T, 1]) one can find in the literature fixed point results for uniformly $k$-lipschitzian semigroups of mappings.

In particular, a fixed point free $\frac{\pi}{2}$-uniformly lipschitzian selfmapping of the positive part $B_{2}^{+}$of the closed unit ball $B_{2}$ of the classical real Hilbert space $\ell_{2}$ is well known. This example is often known as the Baillon mapping (see [T] and [G-K]).

In a recent paper $([\overline{\mathrm{Kc}}), \mathrm{T}$. Kukzumov implicitly asks whether (or not) there exists a renorming $|\cdot|$ of $\ell_{2}$ that makes the Baillon mapping $|\cdot|$-nonexpansive on $B_{2}^{+}$. If this were the case, as $B_{2}^{+}$is a closed bounded convex subset of the superreflexive space $\left(\ell_{2},|\cdot|\right)$, a longstanding open question in Metric Fixed Point Theory would have a negative answer. (See [G-K] for more information.)

The question raised on $B_{2}^{+}$by Kuczumow is deep and natural. First, because a $|\cdot|$-nonexpansive mapping $T: B_{2}^{+} \rightarrow B_{2}^{+}$is always uniformly lipschitzian with respect to the Euclidean norm. Second, because the set $B_{2}^{+}$under consideration could be at least as relevant as the norm, in order to obtain reductions of the Lipschitz constant of a mapping $T: B_{2}^{+} \rightarrow B_{2}^{+}$. To illustrate this, we can regard the following celebrated example due to T.C. Lim ([Li $)$.

He defined the mapping $\mathcal{T}$ in the classical space $\ell_{1}$ by

$$
\mathcal{T}\left(\left(x_{n}\right)\right):=\left(1-\sum_{n=1}^{\infty} x_{n}, x_{1}, x_{2}, \ldots\right) .
$$

Received by the editors November 17, 2000.

2000 Mathematics Subject Classification. Primary 47H10; Secondary 46B03, 46B20.

Key words and phrases. Uniformly lipschitzian mappings, Lipschitz constant, fixed points.

The author was supported in part by a Grant from MCYT, BFM2000-0344-C02-02.

(C)2001 American Mathematical Society 
It is easy to check that $\mathcal{T}$ has the Lipschitz constant 2 on the closed unit ball $B_{1}$ of $\ell_{1}$ with respect to the standard norm of $\ell_{1}$. Moreover, this value 2 is the minimum for the Lipschitz constant of $\mathcal{T}$ on $B_{1}$ under equivalent renormings of $\ell_{1}$. This follows from a straightforward application of Theorem 4 in [Ll].

Nevertheless, Lim showed in [Li] that on $B_{1}^{+}:=\left\{x \in \ell_{1}^{+}: \sum_{n=1}^{\infty} x_{n} \leq 1\right\}$ (the restriction of) $\mathcal{T}$ is an isometry (and hence nonexpansive) with respect to the equivalent norm $\|x\|:=\max \left\{\left\|x^{+}\right\|_{1},\left\|x^{-}\right\|_{1}\right\}$. (Here $x^{+}, x^{-}$are respectively the positive and the negative part of $x \in \ell_{1}$.)

Furthermore, P.N. Dowling and C. Lennard (D-L]) recently defined another equivalent norm on $\ell_{1}$, namely $|x|:=\left|\sum_{n=1}^{\infty} x_{n}\right|+\sum_{n=1}^{\infty}\left|x_{n}\right|$, in such a way that $\mathcal{T}$ is also an $|\cdot|$-isometry, again on $B_{1}^{+}$.

These facts show that dramatic reductions of the Lipschitz constant of a mapping $T$ are possible by renormings of the underlying space, mainly when $T$ is restricted to a suitable $T$-invariant subset of its domain.

In this note we will find lower bounds for the Lipschitz constant $k$ of two uniformly $k$-lipschitzian selfmappings of $B_{2}^{+}$after equivalent renormings of $\ell_{2}$. One of them gives a negative answer to the above-mentioned question raised by Kuczumow.

\section{A RIGID LipsChitz CONSTANT}

Our main result deals with the Baillon mapping $T: B_{2} \rightarrow B_{2}$ given by

$$
T(x)= \begin{cases}\cos \left(\frac{\pi}{2}\|x\|_{2}\right) e_{1}+\sin \left(\frac{\pi}{2}\|x\|_{2}\right) \frac{1}{\|x\|_{2}} S(x), & x \neq 0 \\ e_{1}, & x=0\end{cases}
$$

where $\|x\|_{2}=\left(\sum_{n=1}^{\infty}\left|x_{n}\right|^{2}\right)^{\frac{1}{2}}, e_{1}:=(1,0, \ldots) \in \ell_{2}$ and $S$ is the right shift operator defined by $S\left(x_{1}, x_{2}, \ldots\right):=\left(0, x_{1}, x_{2}, \ldots\right)$. It is well known that $T$ is a $\|\cdot\|_{2^{-}}$ noncontractive uniformly $\frac{\pi}{2}$-lipschitzian on $B$. (See [B], [T].)

Theorem 2.1. Let $T: B_{2}^{+} \rightarrow B_{2}^{+}$be the Baillon mapping. Let $\|\cdot\|$ be an equivalent norm on $\ell_{2}$. Then for each positive $k<\frac{\pi}{2}$ the mapping $T$ is not uniformly $\|\cdot\|-k$ lipschitzian on $\mathrm{B}^{+}$.

Proof. To obtain a contradiction suppose that there exists an equivalent renorming $\|\cdot\|$ of $\ell_{2}$ for which $T$ is $k$ uniformly lipschitzian.

Let $\mu:=\limsup \left\|e_{n}\right\|>0$, where $\left(e_{n}\right)$ is the standard Schauder basis of $\ell_{2}$. Without loss of generality we may suppose that $\mu=1$. Otherwise, we can consider the norm $\frac{1}{\mu}\|\cdot\|$.

Choose any $\varepsilon>0$ so that $\frac{2}{\pi}(1+\varepsilon) k<1$. There exist $n_{0} \in \mathbb{N}$ such that $\left\|e_{n_{0}}\right\|<$ $1+\varepsilon$.

For all positive integers $n, T^{n}\left(e_{n_{0}}\right)=e_{n+n_{0}}$. Moreover, for each $\lambda \in(0,1)$,

$$
T\left(\lambda e_{n_{0}}\right)=\cos \left(\frac{\pi}{2} \lambda\right) e_{1}+\sin \left(\frac{\pi}{2} \lambda\right) \frac{1}{\lambda} S\left(\lambda e_{n_{0}}\right)=\cos \left(\frac{\pi}{2} \lambda\right) e_{1}+\sin \left(\frac{\pi}{2} \lambda\right) e_{n_{0}+1}
$$

and

$$
T^{2}\left(\lambda e_{n_{0}}\right)=S\left(T\left(\lambda e_{n_{0}}\right)\right)=\cos \left(\frac{\pi}{2} \lambda\right) e_{2}+\sin \left(\frac{\pi}{2} \lambda\right) e_{n_{0}+2} .
$$

A simple induction shows that for any positive integer $n$,

$$
T^{n}\left(\lambda e_{n_{0}}\right)=\cos \left(\frac{\pi}{2} \lambda\right) e_{n}+\sin \left(\frac{\pi}{2} \lambda\right) e_{n_{0}+n}
$$


Since $T$ is $k-\|\cdot\|$-uniformly lipschitzian,

$$
\begin{aligned}
\left\|\cos \left(\frac{\pi}{2} \lambda\right) e_{n}-\left(1-\sin \left(\frac{\pi}{2} \lambda\right)\right) e_{n+n_{0}}\right\| & =\left\|T^{n}\left(\lambda e_{n_{0}}\right)-T^{n}\left(e_{n_{0}}\right)\right\| \\
& \leq k\left\|\lambda e_{n_{0}}-e_{n_{0}}\right\| \\
& =k(1-\lambda)\left\|e_{n_{0}}\right\| \\
& <k(1-\lambda)(1+\varepsilon) .
\end{aligned}
$$

Thus,

$$
\frac{\cos \left(\frac{\pi}{2} \lambda\right)}{1-\lambda}\left\|e_{n}\right\|-\frac{1-\sin \left(\frac{\pi}{2} \lambda\right)}{1-\lambda}\left\|e_{n+n_{0}}\right\| \leq k(1+\varepsilon),
$$

and letting $\lambda \rightarrow 1^{-}$we have

$$
\frac{\pi}{2}\left\|e_{n}\right\| \leq k(1+\varepsilon)
$$

From this, we finally obtain

$$
1=\underset{n}{\lim \sup }\left\|e_{n}\right\| \leq \frac{2}{\pi} k(1+\varepsilon)<1,
$$

a contradiction.

Nonexpansive mappings are uniformly 1-lipschitzian. Combining this fact with the above Theorem, we can answer the Kuczumow question in the negative.

Corollary 2.1. The mapping $T$ cannot be nonexpansive on $B_{2}^{+}$with respect to any equivalent renorming of $\ell_{2}$.

\section{The Goebel-Kirk-Thele mapping}

It is quite natural to extend Kuczumow's question to other well-known fixed point free uniformly lipschitzian selfmappings of $B_{2}^{+}$.

Perhaps the first example of a Lipschitzian fixed point free mapping $\varphi: B_{2} \rightarrow B_{2}$ was given by Kakutani in 1943 (see [K]) as follows:

$$
\varphi(x):=\frac{1}{2}\left(1-\|x\|_{2}\right) e_{1}+S(x) .
$$

Kakutani's construction can be generalized to the family of mappings

$$
K_{\varepsilon}(x):=\varepsilon\left(1-\|x\|_{2}\right) e_{1}+S(x),
$$

for $0<\varepsilon \leq 1$. In fact, $K_{\varepsilon}$ maps $B_{2}^{+}$into itself and it has the $\|\cdot\|_{2}$-Lipschitz constant $\sqrt{1+\varepsilon^{2}}$.

A further development of Kakutani's example is due to K. Goebel, W.A. Kirk and R.L. Thele (see G-K-T, 2 ). It is the mapping $G: B_{2}^{+} \rightarrow B_{2}^{+}$given by

$$
G(x):=\frac{1}{\left\|K_{1}(x)\right\|_{2}} K_{1}(x) .
$$

One can easily check that $G$ is uniformly-2-lipschitzian on $B$. The following result shows that this uniform Lipschitz constant 2 could be smaller after a renorming, but not too much smaller.

Theorem 3.1. Let $G: B_{2}^{+} \rightarrow B_{2}^{+}$be the Goebel-Kirk-Thele mapping. Let $\|\cdot\|$ be an equivalent renorming on $\ell_{2}$. If $k$ is the uniform Lipschitz constant of the semigroup of iterates $\left\{G^{n}: n \in \mathbb{N}\right\}$ with respect to the norm $\|\cdot\|$, then $k \geq \sqrt{2}$. 
Proof. Let $\left(e_{n}\right)$ be the standard Schauder basis of $\ell_{2}$. Let $\mu:=\lim \sup \left\|e_{n}\right\|>0$. Fix a positive integer $m$ and $\varepsilon \in\left(0, \frac{1}{2}\right)$. A simple induction shows that, for any positive integer $n$,

$$
\begin{aligned}
& G^{n}\left(\left(\frac{1}{2}+\varepsilon\right) e_{m}\right)=\frac{1}{\sqrt{\left(\frac{1}{2}-\varepsilon\right)^{2}+\left(\frac{1}{2}+\varepsilon\right)^{2}}}\left[\left(\frac{1}{2}-\varepsilon\right) e_{n}+\left(\frac{1}{2}+\varepsilon\right) e_{m+n}\right], \\
& G^{n}\left(\left(\frac{1}{2}-\varepsilon\right) e_{m}\right)=\frac{1}{\sqrt{\left(\frac{1}{2}-\varepsilon\right)^{2}+\left(\frac{1}{2}+\varepsilon\right)^{2}}}\left[\left(\frac{1}{2}+\varepsilon\right) e_{n}+\left(\frac{1}{2}-\varepsilon\right) e_{m+n}\right] .
\end{aligned}
$$

Since $G$ is uniformly $k$-lipschitzian with respect to the norm $\|\cdot\|$ on $B^{+}$, for any positive integer $n$,

$$
\begin{aligned}
\frac{2 \varepsilon\left\|e_{m+n}-e_{n}\right\|}{\sqrt{\left(\frac{1}{2}-\varepsilon\right)^{2}+\left(\frac{1}{2}+\varepsilon\right)^{2}}} & =\left\|G^{n}\left(\left(\frac{1}{2}+\varepsilon\right) e_{m}\right)-G^{n}\left(\left(\frac{1}{2}-\varepsilon\right) e_{m}\right)\right\|, \\
& \leq 2 \varepsilon k\left\|e_{m}\right\|
\end{aligned}
$$

that is,

$$
\frac{1}{\sqrt{\left(\frac{1}{2}-\varepsilon\right)^{2}+\left(\frac{1}{2}+\varepsilon\right)^{2}}}\left\|e_{m+n}-e_{n}\right\| \leq k\left\|e_{m}\right\| .
$$

Letting $\varepsilon \rightarrow 0^{+}$we obtain, for any positive integers $m$ and $n$,

$$
\sqrt{2}\left\|e_{m+n}-e_{n}\right\| \leq k\left\|e_{m}\right\| \text {. }
$$

From this, as the sequence $\left(e_{m+n}-e_{n}\right)_{m \geq 1}$ converges weakly to $-e_{n}$, we have

$$
\sqrt{2}\left\|-e_{n}\right\| \leq \liminf _{m} \sqrt{2}\left\|e_{m+n}-e_{n}\right\| \leq k \limsup _{m}\left\|e_{m}\right\|=k \mu .
$$

As this inequality holds for any positive integer $n$, we finally derive that

$$
\sqrt{2} \mu=\sqrt{2} \limsup _{n}\left\|-e_{n}\right\| \leq k \mu,
$$

which yields the conclusion.

Corollary 3.1. The mapping $G$ cannot be nonexpansive on $B_{2}^{+}$with respect to any equivalent renorming of $\ell_{2}$.

\section{FURTHER REMARKS}

4.1. About the Kakutani mapping. We include here a result about the Kakutani mapping which may be well known, but whose proof is not easily found in the literature. We can see that the iterates of this mapping do not admit a uniform Lipschitz constant on $B_{2}^{+}$.

Theorem 4.1. The mapping $K_{1}: B_{2}^{+} \rightarrow B_{2}^{+}$given by

$$
K_{1}(x)=\left(1-\|x\|_{2}\right) e_{1}+S(x)
$$

is not uniformly lipschitzcian on $B_{2}^{+}$.

Proof. For any $x \in B_{2}^{+},\left\|K_{1}(x)\right\|_{2} \geq\|x\|_{2}$, that is, the sequence $\left(\left\|K_{1}^{n}(x)\right\|_{2}\right)_{n=1}^{\infty}$ is nondecreasing. Hence, there exists $\ell:=\lim _{n \rightarrow \infty}\left\|K_{1}^{n}(x)\right\|_{2} \leq 1$.

Since

$$
\left.\left\|K_{1}^{n+1}(x)\right\|_{2}^{2}=\left(1-\left\|K_{1}^{n}(x)\right\|_{2}^{2}\right)+\left\|S\left(K_{1}^{n}(x)\right)\right\|_{2}^{2}=\left(1-\left\|K_{1}^{n}(x)\right\|_{2}\right)^{2}+\| K_{1}^{n}(x)\right) \|_{2}^{2},
$$

letting $n \rightarrow \infty$, we obtain $\ell^{2}=(1-\ell)^{2}+\ell^{2} \Rightarrow \ell=1$.

Bearing this in mind, to obtain a contradiction, we suppose that $K_{1}$ is uniformly $k$-lipschitzian on $B_{2}^{+}$. 
Then for any positive integer $n$ and $\lambda \in(0,1)$,

$$
\left\|K_{1}^{n}\left(e_{1}\right)-K_{1}^{n}\left(\lambda e_{1}\right)\right\|_{2} \leq k(1-\lambda),
$$

that is,

$$
\sqrt{\left\|K_{1}^{n}\left(e_{1}\right)\right\|_{2}^{2}+\left\|K_{1}^{n}\left(\lambda e_{1}\right)\right\|_{2}^{2}-2\left\langle K_{1}^{n}\left(e_{1}\right), K_{1}^{n}\left(\lambda e_{1}\right)\right\rangle} \leq k(1-\lambda),
$$

where $\langle\cdot, \cdot\rangle$ stands for the inner product in $\ell_{2}$. As $K_{1}^{n}\left(e_{1}\right)=e_{n+1}$, it is straightforward to check that

$$
\left\langle K_{1}^{n}\left(e_{1}\right), K_{1}^{n}\left(\lambda e_{1}\right)\right\rangle=\lambda .
$$

Thus,

$$
\sqrt{\left\|K_{1}^{n}\left(e_{1}\right)\right\|_{2}^{2}+\left\|K_{1}^{n}\left(\lambda e_{1}\right)\right\|_{2}^{2}-2 \lambda} \leq k(1-\lambda) .
$$

Letting $n \rightarrow \infty$, we obtain

$$
\sqrt{2-2 \lambda} \leq k(1-\lambda)
$$

which yields the inequality

$$
\frac{\sqrt{2}}{\sqrt{1-\lambda}} \leq k
$$

for all $\lambda \in(0,1)$. Taking limits when $\lambda$ tends to $1^{-}$we obtain the desired contradiction.

Corollary 4.1. The mapping $K_{1}$ cannot be nonexpansive on $B_{2}^{+}$with respect to any equivalent norm of $\ell_{2}$.

4.2. In G-J-L the authors showed that it is impossible to find in $\ell_{2}$ an equivalent renorming for which some of the three above mappings $T, G, K_{\varepsilon}$ could attain the Lipschitz constant 1 on $B_{2}$. Corollaries 1-3 contain the same result on $B_{2}^{+}$. One can note that the proofs of $\left[\mathrm{G}-\mathrm{J}-\mathrm{L}\right.$ ] cannot be adapted to $B_{2}^{+}$because this set does not have interior points.

The same happens with the results about lipschitzian mappings in the recent paper [Ll].

\subsection{Questions.}

4.3.1. Is it possible to find a norm on $\ell_{2}$ for which the (exact) uniform Lipschitz constant of $G$ on $B_{2}^{+}$is $\sqrt{2}$ ? In other words, is the bound $\sqrt{2}$ in Theorem 2 sharp?

4.3.2. If $U$ is any of the mappings $K_{\varepsilon}, G, T$, is $B^{+}$a minimal bounded closed convex $U$-invariant set? If the answer is no, it seems interesting to look for one.

\section{REFERENCES}

[B] Baillon, J.B., Quelques aspects de la théorie des points fixes dans les espaces de Banach, I, Séminaire d'Analyse Fonctionelle de l'Ecole Polytecnique, no. VII, 197879. MR 81d:47036

[D-L] Dowling, P.N., Lennard, C.J., Every nonreflexive subspace of $L^{1}[0,1]$ fails the fixed point property, Proc. A. M. S., 125 (1997), 443-446. MR 97d:46034

[G-J-L] García-Falset, J., Jiménez-Melado, A. and Llorens-Fuster, E., Isomorphically expansive mappings in $\ell_{2}$, Proc. A. M. S., 125 (1997), 2633-2636. MR 97j:47079

[G-K] Goebel, K. and Kirk, W.A., Topics in metric fixed point theory, Cambridge Univ. Pres., 1990. MR 92c: 47070 
[G-K-T, 1] Goebel, K., Kirk W.A. and Thele, R.L., A fixed point theorem for mappings whose iterate have uniform Lipschitz constant, Studia Math. XLVII, (1973), 135-140.

[G-K-T, 2] Goebel, K., Kirk W.A. and Thele, R.L., Uniformly Lipschitzian semigroups in Hilbert space, Canad. J. Math. 26 (1974), 1245-1256.

[K] Kakutani, S. Topological Properties of the Unit Sphere of a Hilbert space, Proc. Imp. Acad. Tokyo, 19 (1943), 269-271. MR 7:2521

[Lf] Lifschitz, E.A. Fixed point theorems for operators in strongly convex spaces, Voronez. Gos. Univ. Trudy Mat. Fak., 16 (1975), 23-28. MR 57:17401

[Li] Lim, T.C. Asymptotic centers and nonexpansive mapping in conjugate Banach spaces, Pacific J. of Math, 90 (1), (1980), 135-143. MR 82h:47052

[Ll] Llorens-Fuster, E. Renorming and minimal Lipschitz constants, Nonlinear Analysis, 47 (4), (2001), 2719-2730.

[Kc] Kuczumow, T. Opial's modulus and fixed points of semigroups of mappings Proc. Amer. Math. Soc. 127 (1999), 2671-2678. MR 99m:47064

[T] D. Tingley Noncontractive uniformly Lipschitzian semigroups in Hilbert space, Proc. A. M. S., 92, 3, (1984), 355-361. MR 85k:47015

Departamento de Análisis Matemático, Facultad de Matemáticas, Baton 46100 BurJassot, VALENCIA, Spain

E-mail address: enrique.llorens@uv.es 